\title{
Preferencias individuales y elección social. La inocencia epistémica de las teorías
}

\author{
OBDULIA TORRES GONZÁLEZ \\ Universidad de La Laguna
}

RESUMEN. El enfoque economicista que predomina actualmente en el campo de la elección social tiene consecuencias indeseadas cuando se aplica en las elecciones de los posibles estados sociales, es decir, en las elecciones colectivas no económicas. $\mathrm{La}$ razón fundamental es que el uso del modelo económico proscribe cualquier consideración axiológica en el terreno de la elección, eliminándose de un plumazo, en el diseño de los agentes, las consideraciones éticas y políticas, y cualquier referencia acerca de los valores de los individuos en el proceso de toma de decisiones colectivas.

\section{INTRODUCCIÓN}

La teoría de la elección social hunde sus raíces en la Economía del Bienestar que podemos situar en torno a los años veinte del siglo $\mathrm{xx}, \mathrm{y}$ en lo que tradicionalmente se ha denominado Economía Política. En ella se entrecruzan principios teóricos, axiomas formales; concepciones de la organización social y criterios acerca de cómo y bajo qué normas ha de ser redistribuida la riqueza y diseñadas nuestras instituciones. Estamos, por tanto, en un cruce de caminos entre la economía, la política y la ética.

El fundamento último de esta teoría, su elemento explicativo básico, está en las elecciones de los agentes $\mathrm{y}$, por tanto, en las preferencias individuales. Esto es así porque no puede entenderse la teoría de la elección social fuera de un contexto demo-
ABSTRACT. The economic approach, that actually prevail in the social choice studies, have no want consequence when is used in choice of possible social states. The basic reason is that economic model proscribe any axiological consideration in this field, removing in agent's design, ethics and political consideration and any reference about individual's values in the take of collective decisions process.

crático, donde se entiende que los individuos pueden elegir, que el sistema expresa las preferencias individuales sobre políticas alternativas, y que son los deseos, o preferencias individuales, los que deben determinar las elecciones colectivas.

La teoría no habla sólo de elecciones políticas en el sentido usual del término, es decir, como votaciones en comicios. Este enfoque se plantea como una teoría general acerca de las elecciones colectivas, siempre y cuando estas elecciones se basen en las preferencias de los agentes y pretendan seleccionar la alternativa óptima para ese conjunto de agentes, bien sea a través de la construcción de una función de bienestar social o mediante el diseño de algún mecanismo de votación.

A partir de la segunda mitad del siglo $\mathrm{xx}$ se produce, por parte de los teóri- 
cos de la elección social ${ }^{1}$, un esfuerzo importante destinado a positivizar su propia área de estudio. Esta positivización se produce mediante la introducción del método y los instrumentos de la teoría económica y se basa en el supuesto fundamental de que los individuos que participan en los procesos de elección colectiva son los mismos que actúan en el mercado $y$, sobre todo, bajo las mismas motivaciones. Si hasta ese momento se había supuesto que los individuos que actúan en la vida pública lo hacían impulsados por motivaciones distintas, como, por ejemplo, la búsqueda del bien común, a partir de ese momento asistimos a la entrada en la escena de la elección social del modelo del homo economicus. Esto implica considerar a los actores sociales en cualquier ámbito de elección, sea político, social o económico, como seres egoístas racionales que intentan maximizar su utilidad. En el ámbito económico se define dicha utilidad como una función creciente de la riqueza, mientras en el ámbito de la elección social la encontramos definida como bienestar.

Nuestro objetivo en las páginas que siguen es mostrar que la positivización y el enfoque economicista que predomina actualmente en el campo de la elección social tiene consecuencias indeseadas cuando se aplica en las elecciones de los posibles estados sociales ${ }^{2}$, es decir, en las elecciones colectivas no económicas. La razón fundamental es que el uso del modelo económico proscribe cualquier consideración axiológica en el terreno de la elección, eliminándose de un plumazo, en el diseño de los agentes, las consideraciones éticas y políticas, y cualquier referencia acerca de los valores de los individuos en el proceso de toma de decisiones colectivas. Muchos de los principios evaluativos de los sujetos son eliminados porque entran en contradicción con el modelo planteado acerca de las elecciones de los agentes, mientras otros se introducen por la puerta de atrás. Esto tiene como conse- cuencia un modelo de elección colectiva sesgado que dista mucho de ser ética y políticamente neutro. La reflexión, que planteamos, estará guiada por la idea de que en su trasfondo operan supuestos de naturaleza ético-política y sus objetivos están asociados a valores políticos y éticos concretos $^{3}$. Su aparente inocencia epistémica vela asunciones y tomas de partido que operan en el trasfondo de la teoría. Y esto tiene una importancia fundamental, dado que las explicaciones que proporciona dicha teoría actúan como fundamento en las decisiones de política pública y en el diseño de nuestras instituciones.

En primer lugar, estableceremos el modelo de elección propio de la teoría de la elección social, haciendo especial hincapié no en lo que los axiomas afirman, sino en lo que suponen y excluyen. A continuación veremos, a través de algunos ejemplos concretos de aplicación de la teoría, las consecuencias que su uso plantea. La selección de estos ejemplos viene determinada por el hecho de que son los casos más conspicuos, los que permiten investigar, por un lado, si la pretendida neutralidad axiológica del modelo no es tal, y, por otro, si pretender ignorar el papel que juegan los valores en la toma de decisiones de los individuos, en el marco de la elección social, tiene consecuencias indeseadas en el diseño institucional. Nuestras conclusiones pretenden reflexionar sobre la posibilidad de que siendo el homo economicus el que puebla nuestras teorías, su comportamiento, en el que nadie, por supuesto, se reconoce, acabe siendo sancionado en el mundo real, dándole entidad a una construcción teórica que poco parece tener que ver con la realidad. Nos preocupa fundamentalmente que el diseño de nuestras instituciones y la forma de organizar la vida compartida se realice en función de un individuo definido como un maximizador de utilidad entendida ésta como riqueza neta. 


\section{EL MODELO DE ELECCIÓN INDIVIDUAL}

El modelo de elección que se traslada a la teoría de la elección social proviene de la teoría del comportamiento del consumidor. En este modelo, el agente viene definido por un conjunto de creencias, matemáticamente representadas por una distribución de probabilidad y un conjunto de preferencias, respecto a las posibles opciones a elegir, representadas por una función de utilidad. La teoría afirma que si el agente decisor se comporta racionalmente, elegirá aquel curso de acción, sujeto a las restricciones pertinentes, que conduce al máximo de la función, siempre y cuando ésta posea una serie de propiedades matemáticas previamente especificadas. La explicación de la acción individual dice que el agente ha elegido la opción que maximiza su utilidad. La importancia atribuida a la construcción de funciones de utilidad individual, cuando nos situamos en el campo de la elección social, viene dada por la necesidad de que aquéllas actúen como índices de una función de bienestar social que seleccione el estado social óptimo dentro de los posibles. Igual que en la teoría microeconómica las funciones de utilidad individual describen aquel conjunto de bienes con los cuales, dentro de su restricción presupuestaria, el agente obtiene la máxima satisfacción; la función de bienestar social permitiría seleccionar aquella situación social, acorde a la definición especificada, en la cual los agentes obtienen el máximo bienestar.

Una función de bienestar social no es sino, en definitiva, una forma de comparar la utilidad de los distintos individuos en lo que a elecciones sociales se refiere. $Y$ es precisamente esa comparación de utilidades la que ha hecho que la construcción de funciones de bienestar social haya estado y continúe estando plagada de dificultades ${ }^{4}$. Ello hace que los esfuerzos teóricos se dirijan fundamentalmente a otro modo de agregar preferencias individuales respecto a situaciones sociales: los mecanismos de votación. Son ellos los que nos permiten el paso de preferencias individuales a elecciones sociales. En este sentido las preferencias de los agentes determinan tanto las elecciones individuales como las colectivas.

Preferir implica comparar. Declaramos nuestra preferencia por una acción u objeto sobre otro, por tanto, se establece una relación entre esos objetos determinada por la preferencia del agente. La relación es binaria ${ }^{5}$, es decir, comparamos uno a uno y no muchos a muchos, y como cualquier relación entre miembros de un conjunto posee una serie de propiedades. $\mathrm{Si}$ esas propiedades se cumplen podremos hacer afirmaciones sobre las elecciones individuales. Es esta acepción la que se utiliza en los modelos que pretenden explicar y predecir las elecciones individuales en el mercado y por extensión en la elección social. Veamos a continuación cuáles son las propiedades ${ }^{6}$ que ha de cumplir la relación de preferencias atendiendo más a lo que prohíben y suponen que a lo que afirman y, especialmente, las consecuencias de estas exigencias cuando lo que comparamos son estados o situaciones sociales alternativas.

- La relación ha de ser conexa o completa, es decir, los objetos de elección han de ser comparables de modo que el individuo pueda declarar su preferencia por uno $u$ otro, o bien declararse indiferente.

- La relación ha de ser transitiva de forma que si el individuo prefiere el objeto $x$ al $y$ y el $y$ al $z$, debe preferir $x$ a $z$.

La completud y la transitividad de la ordenación de preferencias presenta problemas relacionados con la cuestión de la adecuación del modelo al comportamiento real de los agentes. Experimentos diseñados ${ }^{7}$ para probar tal adecuación muestran que las conductas de los individuos se alejan en gran medida de lo postulado por la teoría. Pero no nos interesa aquí probar el 
realismo o irrealismo de los supuestos que definen la acción humana en situaciones de elección, sino dar cuenta de cómo la selección de una serie de supuestos condiciona los resultados que es posible obtener en el campo del bienestar social.

La elección ha de ser racional (acorde a la definición) ${ }^{8}$ si $x$ es la alternativa elegida $x$ es la alternativa preferida y, podríamos añadir, la utilidad de $x$ es la más alta dentro del conjunto disponible. Este axioma supone afirmar la identidad entre la elección y la preferencia. Un ejemplo, que viola este axioma, puede ser clarificador: supongamos que $a$ y $b$ son dos marcas de ropa de deporte, siendo $b$ su marca favorita, pero usted acaba de enterarse que $b$ utiliza mano de obra infantil en su manufactura y, en consecuencia, decide comprar $a$. En este caso, la alternativa elegida no es la alternativa preferida, sus valores están operando en el transfondo y su criterio de elección ya no es la maximización de la utilidad. Es este tipo de comportamiento, precisamente, el que proscribe el tercer axioma. Veamos qué ocurre en el campo de la elección social. Hemos de tener presente que en este ámbito se afirma que los individuos maximizan su bienestar y eligen entre situaciones sociales alternativas. Igual que en el terreno estrictamente económico el cumplimiento de este axioma supone afirmar la identidad entre preferencia y elección, por un lado, y entre preferencia y bienestar ${ }^{9}$, por otro. De la misma forma, el cumplimiento del axioma excluye cualquier consideración a principios normativos. Uno de los autores más importantes en el campo de la elección social, Amartya Sen, propone precisamente la necesidad de introducir principios normativos como el de la simpatía y el compromiso en el terreno de la elección. Sen distingue simpatía y compromiso en los siguientes términos: «El primero corresponde al caso en el que el interés por otros afecta a nuestro propio bienestar. Si el conocimiento de que se tortura a otros nos enferma, éste es un caso de simpatía; si no nos enferma pero creemos que es algo malo y estamos dispuestos a hacer algo para detenerlo, éste es un caso de compromiso» ${ }^{10}$. Nos interesa fundamentalmente el principio de compromiso, esto es, cuando los principios de un agente le llevan a seleccionar una situación social donde de hecho su bienestar es menor. El compromiso tiene que ver con la moral en un sentido amplio y es sumamente relevante en muchos aspectos de la elección social, como en la cuestión de los bienes públi$\cos$, su asignación y distribución, en las votaciones estratégicas o en la motivación para el trabajo, áreas de estudio donde la hipótesis del comportamiento maximizador no parece dar cuenta del comportamiento real de los agentes ${ }^{11}$.

Lo importante es que el compromiso introduce una cuña entre preferencia y bienestar, por un lado, y preferencia y elección, por otro ${ }^{12}$. Lo elegido no será ya lo preferido ni lo que aumenta el bienestar personal. Pero el modelo, tal y como ha sido formulado, excluye cualquier referencia a principios normativos. $\mathrm{Ni}$ siquiera permite incluir la simpatía, un principio mucho menos restrictivo, dado que un aumento en el bienestar de otro aumenta mi propio bienestar.

- Los individuos prefieren más a menos. Si existen dos cestas de bienes idénticas, salvo que una de ellas contiene más de un producto que la otra, el agente elegirá aquella que contiene más.

La contrapartida formal de este axioma define la utilidad como una función creciente de la riqueza, es decir, a mayor cantidad de bienes, mayor utilidad. $\mathrm{La}$ cuestión es cómo definimos el bienestar, que es aquello que los individuos maximizan, en el campo de la elección social. Cuando hablamos de bienestar nos referimos, básicamente, a cómo se distribuyen los bienes entre un grupo social determinado. En la teoría microeconómica las preferencias se refieren a cestas de consu- 
mo individual; en el campo del bienestar las preferencias se refieren a asignaciones. Por asignación se entiende una descripción de la cantidad de bienes, ya sean públicos o privados, que obtiene cada individuo en cada situación social posible. En el fondo tanto la utilidad como el bienestar parecen referir a lo mismo: cantidad de bienes en manos de cada persona. Una diferencia fundamental la constituye el hecho de que en las elecciones colectivas las selección de alternativas no se realiza sólo para uno mismo, sino para el grupo social al que se pertenece, las distribuciones de riqueza, trabajo y bienes que definen una situación social incluye al resto de los miembros de la comunidad. La imposibilidad de incluir principios normativos que analizábamos en el apartado anterior excluye la posibilidad de que el bienestar de los otros influya en mi propio bienestar, por tanto, no parece haber mucha diferencia, en lo que a definición formal se refiere, entre bienestar y utilidad. En esa medida la elección se realiza, en uno u otro marco, bajo el supuesto de que el individuo elegirá aquella situación social donde obtenga más beneficio, entendiendo éste como mayor cantidad de bienes, ya sean públicos o privados.

Para que las preferencias puedan ser representadas por una función de utilidad éstas han de ser continuas. Las preferencias de un agente A son continuas si para cada opción $x$ tanto el conjunto superior como el inferior son cerrados. El conjunto superior es el conjunto de opciones $y$ tal que A prefiere $y$ a $x$ o es indiferente entre ambas opciones, el conjunto inferior es igualmente el conjunto de opciones $z$ tal que A prefiere $x$ a $z$ o le es indiferente.

Este axioma parece trivial, pero está a la base de una de las herramientas más potentes del modelo: las curvas de indiferencia, y supone la posibilidad de compensación. Una curva de indiferencia es el lugar geométrico a lo largo del cual el individuo es indiferente entre distintas cantidades de dos bienes. Aumentos en la cantidad de uno de ellos le «compensa» de pérdidas en las cantidades del otro.

La teoría supone que el individuo será indiferente en la siguiente situación: existen dos cestas de bienes, situadas en la misma curva de indiferencia, en las que una de ellas contiene tres libros y dos discos compactos y la otra un libro y cinco discos compactos, el agente prefiere los libros pero un incremento de tres discos le compensa de la pérdida de dos libros.

Este postulado excluye las ordenaciones lexicográficas ${ }^{13}$. En una ordenación de este tipo los objetos de elección se jerarquizan como las palabras en un diccionario En una ordenación lexicográfica el agente siempre preferirá aquella cesta que contiene más cantidad de libros, dado que los libros aparecen en los primeros lugares de la ordenación, y sólo en caso de que contuvieran el mismo número de libros, aquella que contiene más discos. No hay posibilidad de compensar al individuo. La conducta de los agentes en el campo de la política y la elección social parece responder más a una ordenación lexicográfica que a la planteada por el modelo de elección económico. Se supone que habrá cuestiones en las que nunca se podrá compensar al agente como aquellas referidas a derechos y principios. Lo contrario implica suponer que todo es negociable.

- Las preferencias son convexas o la utilidad marginal es decreciente. Esto muy grosso modo quiere decir que la utilidad que proporciona sucesivas unidades del mismo bien crece, pero con una tasa de aumento decreciente.

El primer vaso de agua en el desierto proporciona mayor satisfacción que el décimo. Este postulado justificaba, dentro de la economía del bienestar utilitarista, un sistema de redistribución basado en el principio de maximización de la utilidad social. Pero para poder afirmar que la utilidad marginal es decreciente debemos 
postular utilidades mensurables cardinalmente. La sustitución de la utilidad cardinal por la ordinal significó cambiar la utilidad marginal decreciente por la convexidad de las preferencias lo cual viene a afirmar básicamente lo mismo pero añade la exigencia de que las utilidades de los bienes que entran como argumentos de la función de utilidad sean independientes.

En el caso de la elección social, a la hora de construir una función de bienestar social, tenemos que lo que entran como argumentos de esta función son las utilidades de los distintos individuos que componen la comunidad. La convexidad exige que las utilidades individuales sean independientes, lo cual excluye cualquier influencia del bienestar del otro en mi propio bienestar. Nuevamente se excluye la simpatía y el compromiso.

\section{IMPLICACIONES DEL MODELO EN EL CAMPO DEL BIENESTAR SOCIAL}

En este apartado queremos desarrollar, de forma un poco más exhaustiva, como influye lo hasta aquí señalado en algunas cuestiones tratadas por la teoría de la elección social. Dado que es imposible exponer ésta en toda su complejidad hemos seleccionado algunos casos sobresalientes.

Los puntos señalados pueden ser organizadas en torno a tres ejes. En primer lugar, la equivalencia entre el equilibrio competitivo y el óptimo de Pareto. Tal vez sea ésta una de las cuestiones más importantes sobre las que reflexionar a la hora de cuestionar la pretendida inocencia epistémica de la teoría. En segundo lugar, cómo influyen los supuestos acerca de la naturaleza del ser humano en las explicaciones proporcionadas por la teoría y en el diseño de las instituciones. $Y$, finalmente, cómo influye en ese diseño institucional que la estructura de las preferencias, sin ninguna consideración adicional, sea lo único relevante en la explicación de las elecciones sociales. Todas estas cuestiones se hallan profundamente interrelacionadas.

\section{a) El equilibrio competitivo y el óptimo de Pareto}

Hemos señalado que la teoría de la elección social toma gran parte de su objeto de estudio de la Economía del Bienestar. En este campo, los problemas presentados en torno a la posibilidad de construir una función de bienestar social, hace que se desemboque en el criterio de Pareto, como principio para seleccionar entre situaciones sociales alternativas. Este principio viene a afirmar que debe ser seleccionada aquella situación social donde sea posible mejorar la situación de algún individuo sin empeorar la de algún otro. En una lectura negativa excluye cualquier cambio que implique una pérdida de bienestar aunque sólo sea para un individuo, independientemente de la ganancia asociada a ese cambio, aunque la ganancia afecte a todo el grupo en cuestión. La selección en base a este principio desemboca en un estado óptimo de Pareto, dado que no se puede mejorar la situación de alguien sin empeorar la de algún otro. Estamos hablando, por tanto, de un principio normativo que rige la redistribución social de la riqueza, probablemente el primer paso si queremos hablar de estados o situaciones sociales justas.

El Teorema Fundamental de la Economía del Bienestar afirma la equivalencia entre un equilibrio competitivo y un óptimo de Pareto. Un equilibrio competitivo es una situación donde todos los individuos han maximizado su utilidad, sujeta a sus restricciones presupuestarias, y oferta y demanda han sido igualadas, de forma que nadie quiere o puede intercambiar nada más. Esto será un estado paretianamente óptimo, dado que no se puede mejorar la situación de alguien sin empeorar la de algún otro. 
Para empezar, el equilibrio competitivo, cuando se da, es un resultado del sistema económico, mientras que en el terreno de la política la consecución o no de un estado paretianamente óptimo es un principio normativo. Su pretendida equivalencia conduce a pensar que no nos hemos alejado mucho de la «mano invisible» de A. Smith, como principio normativo de redistribución, donde cada uno, buscando su propio interés, consigue, de forma no intencional, el bien común.

Por otro lado, si las decisiones que afectan a la política pública se toman según este modelo ideal, y eso afirman los manuales, no se da cuenta de que las constricciones que definen el equilibrio económico son tan restrictivas que es poco menos que imposible encontrarlas en contextos reales, con lo que una decisión que sería óptima en el mundo teórico ideal, puede ser más que subóptima en el mundo real.

La equivalencia entre el principio de Pareto y el equilibrio competitivo es otra forma de afirmar la equivalencia ente óptimo de Pareto y máxima eficiencia. El concepto de eficiencia económica viene definido como sigue: «... al dividir una cantidad fija de cualquier cosa entre varios usos competitivos, la asignación eficiente implica que cada unidad del dividendo se asigna en forma tal que la ganancia de su transferencia a un uso será exactamente igual a la pérdida involucrada en su retiro en otro uso» ${ }^{14}$. Esto no es más que la igualación de las tasas marginales de sustitución que sirven de base al equilibrio general competitivo y, como ya se señaló, una situación de equilibrio general es un óptimo de Pareto, el criterio decisor para las medidas de política económica. Pero tal vez la estrategia no haya sido establecer las condiciones por las que un equilibrio competitivo (como resultado) se reconozca en el mundo real, sino forzar la realidad para conseguir las pretendidas eficiencias. «Por esa vía, un producto que arrancaba con intención explicativa, se convierte en una "utopía racional". Lo único que había que hacer era convertir los supuestos de la teoría, en las metas a conseguir. Era otro modo de empezar a hablar de fines» 15 .

En esta perspectiva, la teoría se torna normativa $y$, por tanto, debe ser evaluada según otros criterios.

\section{b) $E l$ homo economicus}

Existen una serie de supuestos implícitos en la concepción de ser humano, como maximizadores de riqueza neta, que tienen consecuencias relevantes en el ámbito de la elección social. Parte de estos supuestos son: que los hombres siempre prefieren más a menos riqueza, que la utilidad de cada individuo depende única y exclusivamente de su consumo particular, que las preferencias son arquimédicas $\mathrm{y}$, sobre todo, que los seres humanos son económicamente eficientes en sus elecciones.

Postular que los seres humanos siempre prefieren más a menos parece un axioma evidente de la conducta humana. Veámoslo ahora a través de un ejemplo de F. Ovejero, donde además se cuestiona la idoneidad del principio de Pareto. «En principio, el paso de una distribución de la renta entre dos individuos de $\mathrm{A}(30,40)$ a B $(60,80)$, en la que el primero pasa de 30 a 60 y el segundo de 40 a 80 , no se puede calificar como mejora paretiana, si no se añade la suposición de que los individuos creen que "más (renta) es preferido (o preferible) a menos". [...] Si en el ejemplo anterior, el supuesto de preferencia de los dos individuos fuera: "es preferible menos (desigualdad) a más", el estado B resultaría peor en sentido paretiano» 16 .

Es decir, el criterio de distribución, en este caso el óptimo de Pareto, depende de un supuesto que no se hace explícito y que, si se sustituye, cambia la asignación final. La cuestión es que la utilidad es una 
función creciente de la riqueza, como señalamos, ésta es la contrapartida teórica de «los seres humanos prefieren más a menos», y éste es un postulado fundamental de la teoría que no puede ser alterado sin modificar la misma.

Tenemos, por otro lado, el supuesto, esta vez explícitamente establecido, de que la utilidad de cada individuo depende únicamente de su consumo particular. Lo cual implica que las funciones de utilidad son independientes unas de otras. Esto es un requisito necesario para determinar un estado de equilibrio, y, por tanto, un óptimo de Pareto, bajo los supuestos de la teoría. Paradójicamente, J. Buchanan, uno de los mayores defensores de la aplicación del modelo económico al ámbito de las elecciones políticas, sostiene una diferencia cuando el individuo actúa en el mercado y cuando actúa en el contexto de la elección social. «Parece probable que el individuo representativo actuará de acuerdo con una escala diferente de preferencias cuando comprende que está eligiendo para el grupo y no para sí mismo» ${ }^{17}$. Esto tiene que ver con lo que podemos denominar de alguna manera la intencionalidad de los procesos. En el mercado, una vez que el consumidor ha maximizado su utilidad y está en equilibrio, hay una asignación eficiente de recursos, la demanda global se iguala a la oferta global, se cumple el objetivo maximizador - pues no habrán reasignaciones del gasto capaces de aumentar la satisfacción total- - y se alcanza un equilibrio en el intercambio. Pero todo ello ocurre, por así decirlo, de espaldas al sujeto. Su objetivo no es la eficiencia total del sistema, ni obtener una situación social dada; su único objetivo es satisfacer sus preferencias o sus necesidades. Mientras que en una situación de elección social sí hay una intencionalidad sobre lo global, sí se piensa en el resultado final y éste es intencionalmente considerado. Según Buchanan ${ }^{18}$, este sentido de participación social ejercería importantes efectos en la conducta individual, ya que el individuo actuaría con una escala de preferencias distintas según la acción se produzca en uno $u$ otro contexto. Las razones aducidas para esta argumentación serían: en primer lugar, la mayor probabilidad de la influencia de sus valores en la ordenación de las alternativas. En segundo lugar, que en el mercado no hay interacción específica entre los individuos, en el sentido de que su acción no se ve directamente influida por la conducta de los otros $^{19}$, ya que éstos son tomados como parámetros, no como variables que determinen la posición del individuo, que es lo que ocurre en el contexto de lo social. Se está admitiendo, por tanto, que los valores individuales son significativos a la hora de realizar la elección, y que esto constituye una diferencia en la elección entre ambos contextos. Se trata, fundamentalmente, de que la función de utilidad ya no estará compuesta, única y exclusivamente, por el beneficio derivado del consumo o la posesión de bienes, sino por «algo más». Ese «algo más» sería la utilidad del resto de los agentes que constituyen la comunidad. Pese a todo, desde los supuestos teóricos, se continúa manteniendo que los preferencias son independientes y que dependen únicamente de la cantidad de bienes en manos de cada individuo. En resumen, en las elecciones sociales el bienestar del individuo no vendría dado exclusivamente por su consumo particular, habría que establecer de alguna manera la forma en que las funciones de utilidad son interdependientes, pero esta interdependencia viene explícitamente prohibida por las herramientas matemáticas utilizadas 20 .

El supuesto de preferencias arquimédicas, implícito en la posibilidad de cons trucción de curvas de indiferencia, se refiere a que siempre es posible compensar al individuo de pérdidas en las unidades de un bien con aumentos en las unidades de otro bien. La cuestión es: ¿es 
posible esta compensación en todos los contextos?

Imaginemos que debemos establecer la compensación que se otorgará a un individuo por trabajar en una industria de riesgo ${ }^{21}$. Su utilidad esperada, $p U(c)$, depende únicamente de su probabilidad de supervivencia y de su consumo, estas serían las variables relevantes aquí. Este tipo de análisis coste-beneficio o riesgo-beneficio, es una forma de introducir la noción de Pareto en los análisis de política económica. Se supone que se pregunta a los afectados qué cantidad de dinero estarían dispuestos a pagar por la implementación de una medida política determinada (caso de que fuera considerada deseable por los agentes), o en cuánto fijarían la compensación a recibir si la medida política fuera finalmente implantada (caso de que fuera considerada indeseable). Esa medida política sería una mejora de Pareto si los ganadores pudieran compensar a los perdedores y todavía obtuvieran algo para sí mismos.

Estamos suponiendo, por un lado, que la compensación es posible; por otro, que los individuos son capaces de estimar $p$ y $c$ de forma que $U$ se mantenga constante $\mathrm{y}$, además, se haga máxima; y, finalmente, que serán los aumentos de $c$ estimados los que sirvan de compensación, independientemente del grupo de sujetos que se esté considerando.

«Que a determinados individuos se les pueda compensar por vivir cerca de una central nuclear, que estén de acuerdo en aceptar riesgos y enfermedades, sólo nos habla de su percepción, de su miseria: cuanto más pobres sean más fácilmente aceptarán. Desde el punto de vista de la percepción subjetiva, los pobres del Tercer Mundo que están dispuestos a morir de leucemia o a trabajar en industrias químicas arriesgando su vida a cambio de un salario se sentirán compensados» ${ }^{22}$. Es evidente que la pregunta por la posibilidad de la compensación ha de ser complemen- tada por la pregunta acerca de la justicia de la misma. Una dificultad adicional refiere a la cuestión de si los individuos poseen las habilidades computacionales necesarias para realizar tales cálculos ${ }^{23}$.

\section{c) La autonomía de las preferencias}

Es una premisa fundamental que las elecciones públicas o colectivas dependen única y exclusivamente de las preferencias individuales. A este nivel, sólo se contemplan los métodos de agregación de dichas preferencias que, aunque poseen valores implícitos, toman las preferencias como dadas. Todo ello fundamentado en el principio de soberanía individual, es decir, en la defensa de la libertad individual; son los deseos de los ciudadanos los que deben determinar las elecciones colectivas. En este sentido, las preferencias son la expresión libre de los deseos. Pero hay multitud de fenómenos que llevan a pensar que las preferencias no son tan libres ni tan autónomas y ello tiene consecuencias importantes en el diseño institucional. Veámoslo a través de dos ejemplos, uno aplicado a la teoría del crimen y otro a los modelos de distribución de bienes bajo regla de mayoría.

Muchas veces, las preferencias son el fruto de un ajuste a la realidad, con el propósito de reducir la disonancia entre nuestros deseos y un mundo que no los satisface. La disonancia se reduce juzgando indeseable lo que no se puede obtener, como es el caso de la zorra y las uvas ${ }^{23}$.

No tener en cuenta que las preferencias, muchas veces, son adaptativas, puede tener consecuencias importantes cuando aplicamos la teoría fuera del ámbito estrictamente económico. G. Tullock aplica la teoría microeconómica a la teoría del crimen. Según él: «La mayoría de los economistas que piensan seriamente en el problema del crimen, inmediatamente llegan a la conclusión de que el castigo, en efec- 
to, impedirá el crimen. La razón es perfec tamente simple [...] Si aumentas el costo de algo, menos será consumido. Así, si incrementas el costo de cometer un crimen habrá menos crímenes» ${ }^{25}$.

Esto parece el argumento típico que justifica, pongamos por caso, la pena de muerte o instituciones penitenciarias más interesadas en castigar que en reinsertar. Pero la cuestión es que estudios en reducción de disonancia cognitiva, muestran que lo anterior no es exactamente así. Si los castigos son menos severos, los sujetos necesitan crear una justificación interna para obedecer las leyes, en esa medida, la automotivación es un factor clave en la reducción del crimen y éste debería disminuir con la severidad del castigo ${ }^{26}$. Esto parece un ejemplo claro de cómo la utilización del modelo de agente económico en cualquier ámbito de elección, en este caso cometer o no cometer un crimen, tiene consecuencias en el diseño institucional. Si realmente creemos que los individuos realizan un análisis coste-beneficio y seleccionan aquella alternativa que les proporciona mayor utilidad, parece natural suponer que un aumento en el coste, penas de cárcel más severas, contribuirán a decantar su elección y no cometerá crímenes. Con lo cual el diseño y los principios que rigen las instituciones penitenciarias será uno. Si creemos que con castigos menos severos los individuos crean una automotivación para obedecer la ley y el crimen se reduce con la disminución de la severidad del castigo, tendremos otro diseño institucional. De esta forma el modelo ideal que se toma tiene consecuencias en la organización práctica de nuestra sociedad.

Para nuestro siguiente caso recurriremos a un ejemplo: supongamos que vamos a dividir un pastel entre tres individuos, y que serán esos mismos individuos quienes, a través de una votación bajo regla de mayoría decidan qué porción del pastel tocará a cada uno. La paradoja es que se necesitan, como mínimo dos individuos para poder tomar una decisión, dado que ninguno de ellos podrá decidir nada si no cuenta con el apoyo de otro miembro del grupo, pero, por otro lado, sólo son necesarios dos individuos para poder tomar una decisión. El conjunto de posibles soluciones, es decir, de imputaciones estables será: $(1 / 2,0,1 / 2),(1 / 2,1 / 2,0)$ y $(0,1 / 2$, 1/2). En la primera solución los individuos 1 y 3 se reparten a medias el pastel y el individuo 2 se queda $\sin$ nada, en la solución dos es el individuo 3 el que no obtiene nada y en la tercera es el 1 el que queda desposeído ${ }^{27}$. Los amantes de la justicia y la equidad pueden pensar, llegados a este punto, que la solución «debería» ser ( $1 / 3$, $1 / 3,1 / 3$ ), donde el pastel se reparte en porciones iguales entre los tres individuos, lo que se denomina una imputación equitativa, pero recuérdese que la partición se realiza bajo regla de mayoría, y se necesita únicamente el acuerdo de esa mayoría para decidir los términos del reparto; por tanto, esta solución equitativa es dominada por cualquiera de las imputaciones antes descritas que se encuentran en el conjunto de solución y que implican un reparto simétrico entre los miembros de la coalición. Tal como afirma Buchanan: «Sobre la hipótesis de la maximización de la utilidad individual [...], la imputación equitativa nunca sería elegida» ${ }^{28}$. El ejemplo del pastel puede parecer trivial, pero ha de tenerse en cuenta que una imputación es lo mismo que una asignación, en el sentido de una descripción de la clase y cantidad de bienes en manos de cada individuo y en qué términos se va a llevar a cabo esa distribución de bienes teniendo sólo en cuenta las elecciones de los individuos.

Cada una de las cuestiones señaladas son merecedoras de un artículo en profundidad. Su análisis en estas páginas ha sido, por razones obvias, necesariamente breve. Pero creemos que es suficiente para inducir a la reflexión sobre la idoneidad de la aplicación de estos modelos por mucho 
que respondan a los desiderata del método científico.

El modelo de agente económico que hemos descrito ha sido cuestionado, desde el mismo ámbito económico, básicamente por su falta de adecuación al comportamiento real de los agentes. Pero lo que hemos querido mostrar a lo largo de estas páginas es que su exportación al campo de la elección social es sumamente problemática, dado que, en primer lugar, proscribe cualquier referencia a los principios y valores de los agentes, en un campo en el que, creemos, éstos deben jugar un papel fundamental. En segundo lugar, introduce, de forma sesgada, valores propios de una concepción política determinada, léase individualismo, eficiencia, etc., y, en tercer lugar, su aplicación tiene consecuencias no intencionales en el diseño institucional y la instauración de las políticas públicas.

Como se señaló en el comienzo de estas páginas, la teoría de la elección social es una teoría que presenta características especiales, dado que las explicaciones que proporciona actúan como fundamento en las decisiones de política pública y en el diseño de nuestras instituciones. Si la teo- ría de la elección social no da cuenta de forma adecuada del paso de las preferencias individuales a las sociales, lo cual es el fundamento teórico básico para su uso, se produce un lapso entre el diseño institucional, la política pública y las motivaciones en las que aparentemente está fundamentado.

Los supuestos teóricos y, sobre todo, sus consecuencias han de hacerse explícitos, sólo sobre esa información pueden los individuos realizar elecciones informadas acerca de la organización social. Las soluciones a la teoría de la elección social exceden el terreno de los axiomas y principios teóricos para adentrarse en el de las asunciones de fondo acerca de la elección, la racionalidad, los valores y principios normativos y las características de los actores sociales. La propuesta es que la teoría de la elección social ha de descansar sobre algo más que el algoritmo de la maximización de la utilidad, que la dimensión ética ha ser introducida si no queremos correr el riesgo de que situándonos, dentro del espectro, en la peor de las conductas posibles acabemos diseñando el peor de los mundos posibles.
1 El término no es descriptivamente adecuado, ya que si hemos de otorgar una partida de nacimiento a la teoría de la elección social, ésta ha de ser en 1951 con la obra de K. J. Arrow, Elección social y valores individuales, Madrid, Instituto de Estudios Fiscales, 1974. Pero sí existía antes de esta fecha un trabajo teórico importante fundamentalmente dirigido a la obtención de una función de bienestar social que permitiera seleccionar la mejor situación social posible en base a funciones de bienestar individuales, en suma, cómo pasar de preferencias individuales a elecciones sociales.

${ }^{2}$ Los objetos de elección dentro de la teoría económica estricta son cestas o bienes de consumo, mientras en la teoría de la elección social son situaciones o estados sociales alternativos: «La definición más precisa de una situación social sería una descrip- ción completa de la cantidad de cada tipo de bienes en manos de cada individuo, la cantidad de trabajo que será aportado por cada individuo, la cantidad de cada factor de producción invertido en cada tipo de actividad productiva, y las cantidades de los diversos tipos de actividades colectivas, tales como servicios municipales, actividades diplomáticas y «su continuación por otros medios» y la erección de estatuas a hombres famosos». K. J. Arrow, Elección social y valores individuales, op. cit., p. 77.

${ }^{3}$ Léase individualismo, eficiencia, etc.

4 Véanse especialmente los trabajos de Bergson y Samuelson y posteriormente los intentos de J. Harsanyi. A. Bergson, «Una nueva formulación de ciertos aspectos de la economía del bienestarm, en K. J. Arrow y T. Scitovski (comps.), Ensayos sobre economía del bienestar, México, Fondo de Cultura Económica, 
1974; P. Samuelson, «La teoría pura del gasto público», en K. J. Arrow y T. Scitovski (comps.), op. cit., y J. Harsanyi, Essays on Ethics, Social Behaviour and Scientific Explanation, Dordrecht, Reidel, 1976.

${ }^{5}$ Las relaciones binarias que se establecen serían las siguientes:

$x R x^{\prime}$ se lee $x$ es preferida o indiferente a $x^{\prime}$; también puede ser leída $x$ es al menos tan buena como $x$. $x P x^{\prime}$ se lee $x$ es preferida a $x^{\prime}$.

$x I x$ ' se lee $x$ es indiferente a $x$,

6 No expondremos la forma matemática de los axiomas. Pueden encontrarse en cuạlquier manual de microeconomía. Son especialmente claros en su exposición: H. A. Green, La teoría del consumidor, Madrid, Alianza, 1986. J. C. Zapatero, Lecciones de microeconomía, Madrid, Nerea, 1987. H. R. Varian, Microeconomía intermedia, Barcelona, Bosch, 1993.

7 Véase, por ejemplo, D. Kahneman y A. Tversky, «Prospect Theory: An Analysis of Decision under Risk», Econometrica, núm. 47, 1979, pp. 263-291. El artículo describe varios estudios empíricos donde los axiomas de la teoría de la utilidad esperada son violados por las elecciones de los sujetos, se argumenta que dicha teoría no es un modelo descriptivo adecuado de la conducta de los sujetos y se propone una teoría alternativa.

${ }^{8} \mathrm{La}$ denominación del axioma es nomenclatura común en los manuales de microeconomía, no se debe confundir con las propuestas de los teóricos de la elección racional de racionalidad como consistencia, coherencia o cualquier otra acepción.

${ }^{9}$ Lo que se elige es lo que se prefiere y lo que se prefiere es lo que proporciona el máximo bienestar.

10 A. Sen, «Los tontos racionales: una critica de los fundamentos conductistas de la teoría económica», en F. Hahn y M. Hollis, Filosofía y teoría económica, México, Fondo de Cultura Económica, 1986, p. 187.

"Tal vez el caso más claro sea la hipótesis del abstencionismo. Bajo los presupuestos de comportamiento maximizador los individuos no votarían en grandes comicios, dado que la posibilidad de que su voto sea decisivo en el resultado final es ínfimo y a él le supone un costo acudir a votar. Todas las teorías que pretenden dar cuenta de por qué los agentes votan y se informan sobre lo que votan (lo cual supone un costo adicional) recurren a alguna forma de compromiso.

${ }^{12}$ En este sentido, A. Sen afirma: «Este vínculo dual entre elección y preferencia de un lado y preferencia y bienestar de otro es crucial a los aspectos normativos de la teoría del equilibrio general. Todos los resultados importantes en este campo dependen de esta relación entre conducta y bienestar a través de la intermediación de la preferencia». A. K. Sen, «Behaviour and the concept of preference», Economica, vol. XL, núm. 159 , 1973, p. 253. Los aspectos normativos de la teoría del equilibrio general refieren a la optimalidad del equilibrio u óptimo de Pareto en la medida en que un estado de equilibrio produce $\mathrm{e}$ máximo bienestar posible, acorde, claro está, a la definición de óptimo de Pareto.

13 La razón de esta exclusión es matemática no empírica. Con una ordenación lexicográfica es imposible dibujar las curvas de indiferencia de un agente.

14 M. Blaug, Teoría económica en retrospección México, Fondo de Cultura Económica, 1985, p. 377

15 F. Ovejero, Mercado, ética y economia, Barcelona, Icaria, 1994, p. 176

16 Ibid., pp. 76-77.

17 J. Buchanan, Economic: Between Predictive Science and Moral Philosophy, Texas, A \& M University Press, 1987 , p. 187. Hay una contradicción explícita en la obra de Buchanan, ya que, en El cálculo del consenso, escrita junto con G. Tullock, afirma: «Inicialmente, sin embargo, podríamos suponer que el individuo medio o representativo actúa sobre la misma escala de valores tanto cuando participa en una actividad de mercado como en la actividad política». J. Buchanan y G. Tullock, El cálculo del consenso, Madrid, Espasa-Calpe, 1980 p. 45. Buchanan utiliza uno u otro argumento según su conveniencia. Afirma que el individuo actúa sobre la misma escala de valores cuando defiende el uso del enfoque económico en la política bajo el rótulo del argumento de simetría. Y afirma que actúa sobre valores distintos cuando intenta refutar la hipótesis de Arrow de que mercado y voto forman parte de una categoría más general que sería la elección social.

$18 \mathrm{~J}$. Buchanan, «Individual Choice in Voting and the Market», en J. Buchanan, Economic: Between Predictive Science and Moral Philosophy, op. cit.

19 Hay un sentido donde esto sí se produce, son los denominados efecto esnobismo y efecto del carro triunfal.

20 Recuérdese que la convexidad de las preferencias exigía utilidades independientes.

21 Normalmente los datos usados en el análisis riesgo-beneficio, para estimar los incrementos de consumo de un individuo que compense disminuciones en su probabilidad de supervivencia, de forma que la utilidad se mantenga constante, son las diferencias salariales observadas en actividades profesionales de riesgo. Ver K. J. Arrow, «Behaviour under Uncertainty and its Implications for Policy», en D. Bell H. Raiffa y A. Tverski (eds.), Decision Making, Cambridge, Cambridge University Press, 1988, pp. 497 507

22 Subrayado en el original. F. Ovejero, 1994 p. 83.

${ }^{23}$ Para un estudio de las limitaciones computacionales de los agentes véase H. Simon, Models of Man, Nueva York, Wiley and Sons, 1957. «De la racionalidad sustantiva a la procesal», en H. Hanh y M. Hollis (comps.), 1986, pp. 130-171, y Models of Bounded Rationality, Cambridge, The MIT Press, 1997. 
24 J. Elster, Uvas amargas, Barcelona, Península, 1988.

25 G. Tullock, «Does Punishment Deter Crime?», Public Interest, 1974, pp. 104-105, en G. Akerlof y W. Dickens, «The Economic Consecuence of Cognitive Dissonance», American Economic Review, 1982 pp. 307-319.

26 lbid., p. 318
${ }^{27}$ Las herramientas que se utilizan en el estudio de formación de coaliciones dentro de la elección social provienen fundamentalmente de desarrollos en Teoría de Juegos. Las soluciones descritas son las soluciones estables, dado que dominan al resto de las posibles combinaciones.

28 J. Buchanan y G.Tullock, El cálculo del consenso, op. cit. 